\title{
A Simplified SARS-CoV-2 Pseudovirus Neutralization Assay
}

\author{
Gaetano Donofrio ${ }^{1, *}$, Valentina Franceschi ${ }^{1}$ (D), Francesca Macchi ${ }^{1}$, Luca Russo ${ }^{1}$, Anna Rocci ${ }^{2}$,

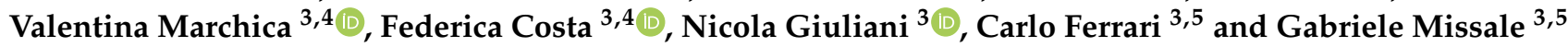 \\ 1 Department of Medical-Veterinary Science, University of Parma, 43126 Parma, Italy; \\ valentina.franceschi@unipr.it (V.F.); francesca.macchi@unipr.it (F.M.); luca.russo@unipr.it (L.R.) \\ 2 Unit of Angiology and Internal Medicine, Azienda Ospedaliero-Universitaria di Parma, 43126 Parma, Italy; \\ arocci@ao.pr.it \\ 3 Department of Medicine and Surgery, University of Parma, 43126 Parma, Italy; \\ valentina.marchica@unipr.it (V.M.); federica.costa@unipr.it (F.C.); nicola.giuliani@unipr.it (N.G.); \\ carlo.ferrari@unipr.it (C.F.); gabriele.missale@unipr.it (G.M.) \\ 4 Unit of Hematology, Azienda Ospedaliero-Universitaria di Parma, 43126 Parma, Italy \\ 5 Laboratory of Viral Immunopathology, Unit of Infectious Diseases and Hepatology, \\ Azienda Ospedaliero-Universitaria di Parma, 43126 Parma, Italy \\ * Correspondence: gaetano.donofrio@unipr.it; Tel.: +39-052-103-2677
}

check for

updates

Citation: Donofrio, G.; Franceschi, V.;

Macchi, F.; Russo, L.; Rocci, A.;

Marchica, V.; Costa, F.; Giuliani, N.;

Ferrari, C.; Missale, G. A Simplified

SARS-CoV-2 Pseudovirus

Neutralization Assay. Vaccines 2021, 9 ,

389. https://doi.org/10.3390/

vaccines 9040389

Academic Editors: Sonia

Navas-Martin and Colleen B. Jonsson

Received: 16 March 2021

Accepted: 13 April 2021

Published: 15 April 2021

Publisher's Note: MDPI stays neutral with regard to jurisdictional claims in published maps and institutional affiliations.

Copyright: (c) 2021 by the authors. Licensee MDPI, Basel, Switzerland. This article is an open access article distributed under the terms and conditions of the Creative Commons Attribution (CC BY) license (https:// creativecommons.org/licenses/by/ $4.0 /)$.
Abstract: COVID-19 is an ongoing pandemic caused by the highly infectious coronavirus SARS$\mathrm{CoV}-2$ that is engaging worldwide scientific research to find a timely and effective eradication strategy. Great efforts have been put into anti-COVID-19 vaccine generation in an effort to protect the world population and block SARS-CoV-2 spread. To validate the protective efficacy of the vaccination campaign and effectively control the pandemic, it is necessary to quantify the induction of neutralizing antibodies by vaccination, as they have been established to be a correlate of protection. In this work, a SARS-CoV-2 pseudovirus neutralization assay, based on a replication-incompetent lentivirus expressing an adapted form of CoV-2 S protein and an ACE2/TMPRSS2 stably expressing cell line, has been minimized in terms of protocol steps without loss of accuracy. The goal of the present simplified neutralization system is to improve SARS-CoV-2 vaccination campaign by means of an easy and accessible approach to be performed in any medical laboratory, maintaining the sensitivity and quantitative reliability of classical serum neutralization assays. Further, this assay can be easily adapted to different coronavirus variants by simply modifying the pseudotyping vector.

Keywords: SARS-CoV-2; COVID-19; neutralizing antibody; pseudovirus; neutralization assay

\section{Introduction}

Coronaviruses, belonging to the family Coronaviridae in the order Nidovirales, are positive-strand RNA viruses with a genome length of between 26 and $32 \mathrm{kbp}$. Several mammalian and avian species can be infected by coronaviruses, which most of the time cause respiratory and/or intestinal disease [1]. Human coronaviruses (HCoVs), such as HCoV-229E, HCoV-OC43, HCoV-NL63, and HKU1 (human coronavirus HCov-HKU1), have long been recognized as major causes of the common cold and are endemic in the human population. Two recent $\mathrm{HCoVs}$, severe acute respiratory syndrome coronavirus (SARS-CoV) and Middle East respiratory syndrome coronavirus (MERS-CoV), emerged in 2002 and 2012, respectively, causing life-threatening disease in humans. A previously unknown coronavirus, named SARS-CoV-2 (CoV-2), was discovered in December 2019 in Wuhan, China, and has been responsible for a pandemic infection, known as coronavirus disease 19 (COVID-19), causing a large number of deaths people worldwide [1] Although great research effort has been made, COVID-19 remains a complex disease showing pathogenetic mechanisms and clinical heterogeneous features that are difficult to understand. A variety of approaches have been employed to develop prophylactic 
and therapeutic measures, including whole inactivated vaccines, subunit vaccines, RNAbased vaccines, viral vectored vaccines [2,3], monoclonal neutralizing antibodies, and fusion inhibitors, most of which were designed to target the CoV-2 spike glycoprotein (S) [4-6]. CoV-2 S, forming homotrimer structures on the viral surface, mediates virus entry to the host cell. S mature structure is formed by S1 and S2 functional subunits: S1 interacts with the angiotensin-converting enzyme 2 (ACE2) cellular receptor, while S2 mediates the viral envelope fusion with the host cell membrane [7-9]. During S1-ACE2 interaction, the host cell surface transmembrane serine protease 2 (TMPRSS2), located next to ACE2 receptor, cleaves the S2 subunit at the S2' amino-terminal portion (815 $\downarrow 816 \mathrm{aa}$; SKR $\downarrow S F I)$, inducing the exposure of fusion peptide hydrophobic domains and the subsequent viral envelope fusion with the host cell membrane [7-9]. Due to its high infectivity and pathogenicity, CoV-2 needs to be handled in biosafety level 3 (BSL-3) specific facilities (https:/ / www.cdc.gov/ coronavirus/2019-ncov/lab/lab-biosafety-guidelines.html, accessed on 5 June 2020) [10], which limits the development of antiviral measures as well as basic and applied studies on the interaction between host cells and CoV-2 and viral attachment and entry mediated by the $\mathrm{S}$ protein. To avoid dealing with infectious CoV-2, several safe, biosafety level 2 (BSL2) pseudovirus-based systems have been developed, mainly based on vesicular stomatitis virus (VSV) [11] or retrovirus (RV) $[12,13]$ vector pseudotyped with CoV-2 S. Although both of them have been shown to be sensitive and reliable, they suffer from being farraginous, time-consuming, and expensive in procedural terms. In the present work, a four-step simplified procedure of $\mathrm{CoV}-2$ pseudovirus neutralization assay was established.

\section{Material and Methods}

\subsection{Plasmids}

ACE2-IRES-TMPRSS2-IRES-Puromycin tricistronic ORF was chemically synthesized and integrated into a lentiviral transfer vector to obtain $\mathrm{pEF} 1 \alpha$-ACE2/TMPRRS2/Puro (Figure S1 for details and full sequence). Similarly, S- $\Delta$ RS-HA ORF (Figure S2) and biscistronic turboGFP-IRES-Luc2 ORF were chemically synthesized and integrated into a lentiviral transfer vector to obtain pLV-CMV-(S- $\triangle$ RS-HA)-IRES-Puro-WPRE (Figure S3) and pLV-EF1 $\alpha$-(turboGFP-IRES-Luc2)-WPRE, respectively. p8.74 packaging, $\mathrm{pREV}$ and pMD2 pseudotyping, and pEGFP-C1 vectors were obtained from Addgene (https:/ / www. addgene.org/, accessed on 3 May 2020).

\subsection{Cells}

Human Embryo Kidney (HEK) 293T (ATCC: CRL-11268) cells were cultured in Eagle's Minimal Essential Medium (EMEM, Gibco; Thermo Fisher Scientific, Carlsbad, CA, USA) containing $1 \mathrm{mM}$ of sodium pyruvate (Gibco, Thermo Fisher Scientific, Carlsbad, CA, USA), $2 \mathrm{mM}$ of L-glutamine (Gibco), $100 \mathrm{IU} / \mathrm{mL}$ of penicillin (Gibco), $100 \mu \mathrm{g} / \mathrm{mL}$ of streptomycin (Sigma-Aldrich, Milano, Italy), and $0.25 \mu \mathrm{g} / \mathrm{mL}$ of amphotericin B (Gibco) (called complete EMEM) supplemented with $10 \%$ fetal bovine serum (FBS, Gibco) and were incubated at $37^{\circ} \mathrm{C}$ and $5 \% \mathrm{CO}_{2}$ in a humidified incubator.

Stably transfected HEK/S- $\triangle$ RS-HA/Puro (HEK/S) and HEK/ACE2/TMPRRS2/Puro cells were obtained by transfecting cells with pLV-CMV-(S- $\triangle$ RS-HA)-IRES-Puro-WPRE or pEF1 $\alpha$-ACE2/TMPRRS2/Puro vectors, respectively. Briefly, subconfluent HEK 293T cells were detached from a T75 $\left(75 \mathrm{~cm}^{2}\right.$ surface area) flask, counted, and electroporated with $20 \mu \mathrm{g}$ of pLV-CMV-(S- $\triangle$ RS-HA)-IRES-Puro-WPRE or pEF1 $\alpha$-ACE2/TMPRRS2/Puro vectors in $600 \mu \mathrm{L}$ of DMEM high glucose (Euroclone, S.p.A, Milan, Italy) without serum at $186 \mathrm{~V}$ and $1500 \mu \mathrm{F}$ in Gene Pulser XCell (Biorad, Milano, Italy).

Electroporated cells were then transferred to new $25 \mathrm{~cm}^{2}$ flasks and fed with complete EMEM with $10 \%$ FBS. Twenty-four hours after the transfection, the medium was changed with fresh complete EMEM with $10 \%$ FBS complemented with $2 \mu \mathrm{g} / \mathrm{mL}$ of puromycin (Millipore Merck Life Science, Milano, Italy). Cells were kept in culture until resistant colonies appeared. Cells were split for more than 40 passages and tested for S or ACE2 expression. 


\subsection{Transient Transfection and Syncytia Formation}

HEK 293T cells were transiently cotransfected with pLV-CMV-(S- $\triangle$ RS-HA)-IRES-PuroWPRE, pEF1 $\alpha$-ACE2/TMPRRS2/Puro and pEGFP-C1 (Clontech, Takara BIO, Mountain View, CA, USA) vectors, with same molar ration (1:1:1), using polyethylenimine (PEI) transfection reagent (Polysciences, Inc. Warrington, PA, USA). Briefly, cells were seeded at $5 \times 10^{5}$ cells/well in 6-well plates and incubated overnight at $37^{\circ} \mathrm{C}$ and $5 \% \mathrm{CO}_{2}$. Cells were then incubated for $6 \mathrm{~h}$ with a transfection mix $(1 \mathrm{~mL})$ containing $3 \mu \mathrm{g}$ of plasmids DNA and PEI (ratio 1:2.5 DNA-PEI) in complete DMEM (Dulbecco's Modified Essential Medium (DMEM, Gibco; Thermo Fisher Scientific, Carlsbad, CA, USA) high glucose (Euroclone) completed with $50 \mu \mathrm{g} / \mathrm{mL}$ of gentamicin (Merk, Darmstadt, Germany) without serum. After incubation, the transfection mix was replaced by fresh complete EMEM and incubated for $24 \mathrm{~h}$ at $37{ }^{\circ} \mathrm{C}$ and $5 \% \mathrm{CO}_{2}$. HEK/ACE2/TMPRRS2/Puro cells were transiently cotransfected with pLV-CMV-(S- $\triangle$ RS-HA)-IRES-Puro-WPRE and pEGFP-C1 as before. Alternatively, HEK/ACE2/TMPRRS2/Puro cells were also cocultivated (1:2 ratio) with HEK 293T cells transiently transfected with pLV-CMV-(S- $\triangle$ RS-HA)-IRES-Puro-WPRE and $\mathrm{pEGFP-C1}$. Twenty-four hours after cocultivation, syncytia were observed by inverted fluorescence microscope (Zeiss-Axiovert-S100), and pictures were acquired by digital camera (Zeiss-Axiocam-MRC). HEK/S- $\triangle$ RS-HA/Puro and HEK/ACE2/TMPRRS2/Puro cells were also cocultivated as before to generate syncytia.

\subsection{Western Immunoblotting}

Protein cell extracts were obtained from pLV-CMV-(S- $\triangle$ RS-HA)-IRES-Puro-WPRE, pEF1 $\alpha$-ACE2/TMPRRS2/Puro and pEGFP-C1 stably transfected HEK293T cells by adding $100 \mu \mathrm{L}$ of cell extraction buffer ( $50 \mathrm{mM}$ Tris- $\mathrm{HCl}, 150 \mathrm{mM} \mathrm{NaCl}$, and 1\% NP-40; pH 8). After BCA total protein quantification (Pierce BCA Protein Assay kit, Thermo Fisher Scientific, Carlsbad, CA, USA), cell extracts containing various amounts of total protein were electrophoresed through 10\% SDS-PAGE. Proteins were then transferred to a nylon membrane by electroblotting, and the membrane was incubated with mouse monoclonal antibody anti-HA tag (G036, Abm Inc., Vancouver, BC, Canada) diluted 1:10,000. After washing, the membrane was incubated with a rabbit anti-mouse IgG secondary antibody labeled with horseradish peroxidase, diluted 1:15,000 (A9044, Sigma-Aldrich, Merk, Darmstadt, Germany). For ACE2 detection, the membrane was incubated with rabbit monoclonal antibody anti-humanACE2 (SN0754, Invitrogen, Thermo Fisher Scientific, Carlsbad, CA, USA) diluted 1:5000. After washing, the membrane was incubated with a goat anti-rabbit IgG secondary antibody labeled with horseradish peroxidase, diluted 1:15,000 (A0545, Sigma-Aldrich). Bands were visualized by enhanced chemiluminescence (Clarity Max Western ECL substrate, Bio-Rad, Hercules, CA, USA).

\subsection{Hematoxylin and Eosin Staining}

Flasks of cells containing syncytia were fixed with $4 \%$ paraformaldehyde in PBS and stained with hematoxylin and eosin standard method.

\subsection{Pseudovirions Reconstitution}

For reconstituting CoV-2 S pseudovirus, HEK 293T cells were transfected, in $T 175 \mathrm{~cm}^{2}$ flasks, with $25 \mu \mathrm{g}$ of pLV-EF1 $\alpha$-(turboGFP-Luc2)-WPRE transfer vector, $15 \mu \mathrm{g}$ of p8.74 packaging vector, $13 \mu \mathrm{g}$ of $\mathrm{pLV}$-CMV-(S- $\triangle \mathrm{RS}-\mathrm{HA})$-IRES-Puro-WPRE pseudotyping vector (although this is a transfer vector, it can work as a pseudotyping vector too), and $5 \mu \mathrm{g}$ of pREV ( $58 \mu \mathrm{g}$ of total DNA) and were diluted in $3 \mathrm{~mL}$ of complete DMEM (Euroclone) without serum and $145 \mu \mathrm{L}$ of PEI (Polysciences, Inc., Warrington, PA, USA) $(1 \mathrm{mg} / \mathrm{mL}$ in PBS) (ratio 1:2.5 DNA/PEI). After at least $15 \mathrm{~min}$ incubation at room temperature, $4 \times$ volumes of complete DMEM without serum were added, and the transfection solution was transferred to the cell monolayer. After $6 \mathrm{~h}$ of incubation at $37^{\circ} \mathrm{C}$ and $5 \% \mathrm{CO}_{2}$, in a humidified incubator, the transfection mixture was replaced with $25 \mathrm{~mL}$ of fresh complete EMEM supplemented with $10 \% \mathrm{FBS}$ and incubated for $48 \mathrm{~h}$ at $37^{\circ} \mathrm{C}$ and $5 \% \mathrm{CO}_{2}$. The 
flask was then frozen-thawed at $-80{ }^{\circ} \mathrm{C}$; transfected cell supernatant (TCS) containing $\mathrm{S}$ pseudovirus was clarified via centrifugation at $3500 \mathrm{rpm}$ for $5 \mathrm{~min}$ at $4{ }^{\circ} \mathrm{C}$, filtered through a $0.45 \mu \mathrm{m}$ filter (Millipore, Merk, Darmstadt, Germany), aliquoted, tittered by limited dilution, and stored at $-80^{\circ} \mathrm{C}$.

ACE2/TMPRSS2 pseudovirus was prepared as described above by simply substituting pLV-CMV-(S- $\triangle$ RS-HA)-IRES-Puro-WPRE with pEF1 $\alpha$-ACE2/TMPRRS2/Puro; again, although this is a transfer vector, it can work as a pseudotyping vector too.

\subsection{Serum Samples}

Sera were collaboratively provided by the Unit of Infectious Diseases and Hepatology, University Hospital of Parma. The study was approved by the local ethical committee (Comitato Etico Area Vasta Emilia Nord (AVEN), Italy). All participants gave written informed consent to participate in the study.

\subsection{Seroneutralization Assay}

Twenty-five microliters of complete EMEM with 10\% FBS was added to each well of a 96-well clear-flat-bottom white microplate with opaque walls (Greiner Bio-One), and $25 \mu \mathrm{L}$ of each serum was added to the first line of wells (Figure S5). Twenty-five microliters of $S$ pseudovirus preparation (described in Section 2.6 above) diluted in complete EMEM with $10 \%$ FBS (corresponding to $\sim 10^{4}$ relative luciferase units (RLUs); $\sim 3-5 \mu \mathrm{L}$ of the initial preparation) was added to each well and left to incubate at room temperature for $1.5 \mathrm{~h}$. Final volume for each well reached $50 \mu \mathrm{L}$; therefore, the sera dilution was doubled, 1:4-1:81:16-1:32-1:64-1:128-1:256-1:512. Next, $50 \mu \mathrm{L}$ of complete EMEM with 10\% FBS, containing $10^{4} \mathrm{HEK} / \mathrm{ACE} 2 / \mathrm{TMPRRS} 2 /$ Puro cells, was added to each well and left for $60 \mathrm{~h}$ at $37^{\circ} \mathrm{C}$ and $5 \% \mathrm{CO}_{2}$.

Plates were read by adding $25 \mu \mathrm{L}$ of complete EMEM containing luciferin to each well just before the reading of the microplate with the luminometer (Victor, Perkin Elmer). A negative control was established without serum, which was substituted with complete medium. The RLUs were compared and normalized to those derived from wells where pseudovirus was added in the absence of sera (100\%). Neutralization titer 50 (NT50) was expressed as the maximal dilution of the sera where the reduction of the signal is $\geq 50 \%$. Worthy of note, the titer had to be multiplied by 40 because the initial volume of the sera tested was $0.025 \mathrm{~mL}$ and it had to be normalized to $1 \mathrm{~mL}$.

\section{Results and Discussion}

\subsection{Generation of a Sensitive Target Cell Line Simultaneously Expressing ACE2 and TMPRRS2}

As ACE2 and TMPRSS2 are the main dependency factors for SARS-CoV-2 (CoV-2) attachment and penetration into the host cells [8], a cell line simultaneously expressing ACE2 and TMPRSS2 was generated. For this purpose, a tricistronic expression cassette was constructed. Human ACE2 ORF (GenBank accession number: NM_021804.3), provided with a strong Kozak's sequence to the $5^{\prime}$ end; human TMPRSS2 ORF (GenBank accession number: NM_001135099.1); and the puromycin drug resistance gene ORF were in-tandem positioned and linked by two internal ribosomal entry sites (IRESs) (ACE2IRES-TMPRSS2-IRES-Puromycin). This large tricistronic ORF was chemically synthesized and subcloned downstream to the human elongation factor 1 alpha promoter (EF1 $\alpha)$, upstream of the SV40 polyA signal/site in a pUC plasmid backbone. Thus, pEF1 $\alpha$ ACE2/TMPRRS2/Puro was generated (Figure S1A). pEF1 $\alpha$-ACE2/TMPRRS2/Puro was electroporated in HEK293T cells, and stably transfected cells were obtained by selection with puromycin. As puromycin drug resistance gene ORF is the last to be translated from the mRNA transcribed from pEF1 $\alpha$-ACE2/TMPRRS2/Puro, the puromycin selection of resistant cells (HEK/ACE2/TMPRRS2/Puro) increases the probability of the majority of the cells expressing ACE2 and TMPRSS2. HEK/ACE2/TMPRRS2/Puro cells were kept for over 30 passages without loss of functionality/expression (Figure S1B). Noteworthily, HEK/ACE2/TMPRRS2/Puro cells do not need to be constantly maintained in culture, 
under puromycin selection: a large batch of them can be grown, aliquoted at a suitable concentration, stored at $-80^{\circ} \mathrm{C}$ or in liquid nitrogen, and successively used, when needed, by simply thawing an aliquot of them, without any loss of functionality.

\subsection{Expression of SARS-CoV-2 Spike Glycoprotein in Mammalian Cells}

CoV-2 S is a key factor for SARS-CoV-2 target cell infection [7-9]. Antibodies from COVID-19 convalescent patient sera can block in vitro and in vivo CoV-2 infectivity; thus, they are exploitable for CoV-2 infection diagnosis and for the evaluation of CoV-2 vaccination efficacy (the titer of neutralizing antibody present in a serum is a correlate of protection), as well as for preliminary test molecules able to interfere with S, ACE2, and TMPRRS2 interaction. Before attempting the generation of a lentivirus-based pseudovirus displaying CoV-2 S on its surface, it was indispensable to generate an appropriate expression cassette capable of driving an efficient expression of $S$, accommodated on the pseudovirus envelope surface. The cytoplasmic tails of some CoV S proteins contain an endoplasmic reticulum retrieval signal (ERRS) that can retrieve $S$ proteins from the Golgi to the endoplasmic reticulum (ER). This process is thought to accumulate $S$ proteins at the CoV budding site, the ER-Golgi intermediate compartment (ERGIC), and to facilitate $S$ protein incorporation into virions $[14,15]$. In contrast to coronaviruses, lentiviruses assemble and bud at the cell surface [16]; therefore, truncation of the S-protein cytoplasmic tail may increase cell surface levels and/or enable incorporation by alleviating structural incompatibility of the S-protein cytoplasmic tail and lentivirus proteins.

CoV-2 S ORF sequence (https:/ / www.ncbi.nlm.nih.gov/protein/1791269090) was deprived of its last $57 \mathrm{bp}$, coding for a predicted ERRS (KFDEDDSEPVLKGVKLHYT) [14] and substituted with the hemagglutinin (HA) tag. The so-designed ORF (S- $\Delta$ RS-HA) was human codon usage adapted, with the use of the Jcat codon adaptation tool (http: / / www.jcat.de), to change nucleotide codon composition to a composition based on human genome codon usage. The degeneracy of the genetic code leads to a situation whereby most of the amino acids can be encoded by two to six synonymous codons. The synonymous codons are not equally utilized to encode the amino acids, thus resulting in the phenomenon of codon usage bias. As codon usage bias has been shown to correlate with gene expression level, it has been proposed as an important design parameter for enhancing recombinant protein production in heterologous host expression [17]. The GC content of S- $\Delta$ RS-HA is $37 \%$, and adaptation to the human genome codon usage shifted the GC content from 37\% up to $63 \%$ (Figure S2). Although we did not compare the adapted S- $\triangle$ RS-HA ORF with the unadapted one in terms of expression efficiency, previous studies have shown that GC-rich genes in mammalian cells can be expressed 100-fold more efficiently than their GC-poor counterparts due to increased steady-state mRNA levels [18]. S- $\Delta$ RS-HA was chemically synthesized and integrated into a transfer vector under the transcriptional guide of the immediate early gene promoter of human cytomegalovirus (CMV) and followed by an IRES, the puromycin drug resistance gene, and the woodchuck hepatitis virus (WHP) posttranscriptional regulatory element (WPRE) (pLV-CMV-(S- $\triangle$ RS-HA)-IRES-Puro-WPRE) (Figure $1 \mathrm{~A}$ and Figure S3A for complete sequence).

pLV-CMV-(S- $\triangle$ RS-HA)-IRES-Puro-WPRE transfected cells successfully expressed S$\triangle$ RS-HA protein, as shown by Western immunoblotting (Figure 1B). When HEK/ACE2/TM PRRS2/Puro were transiently cotransfected with pLV-CMV-(S- $\triangle$ RS-HA)-IRES-Puro-WPRE and pEGFP-C1, a construct delivering enhanced green fluorescent protein (EGFP), large syncytia were observed (Figure 1C). This syncytiogenic effect was attributable to the interaction between S, ACE2, and TMPRRS2, which recapitulated the bona fide fusogenic activity of $S$ when it is attached to ACE2, proteolytically activated by TMPRSS2, and regulated by interferon-induced transmembrane proteins (IFITMs) [19,20]. An identical result was obtained when HEK/ACE2/TMPRRS2/Puro cells were cocultivated with pLVCMV-(S- $\triangle$ RS-HA)-IRES-Puro-WPRE and pEGFP-C1 cotransiently transfected HEK293T cells (Figure 1D). Further, syncytia could be reduced or abrogated if pLV-CMV-(S- $\Delta$ RS- 
HA)-IRES-Puro-WPRE transfected HEK/ACE2/TMPRRS2/Puro cells were treated with human COVID-19 convalescent sera containing anti-S antibodies (Figure S3B).
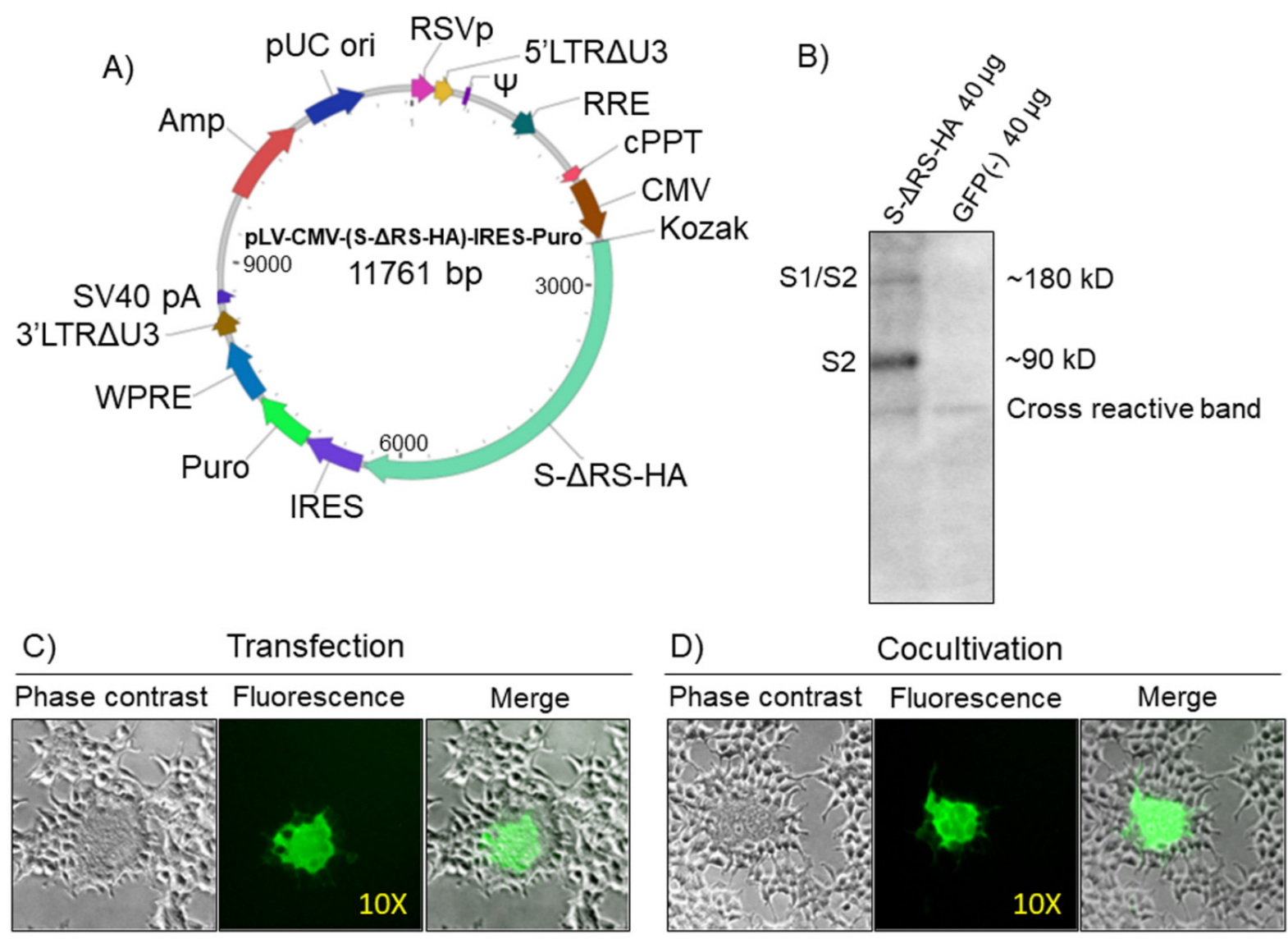

Figure 1. CoV-2 S expression in HEK293T cells. (A) Diagram (not to scale) of pLV-CMV-(S-DRS-HA)-IRES-Puro-WPRE transfer vector, where functional elements of the construct are indicated and highlighted with different colors. RSVp (Rous sarcoma virus enhancer/promoter), 5'LTR $\Delta \mathrm{U} 3$ (truncated HIV-1 5' long terminal repeat), $\Psi$ (HIV-1 packaging signal), RRE (HIV-1 REV response element), cPPT (central polypurine tract), CMV (human cytomegalovirus immediate early enhancer/promoter), Kozak (Kozak's translation initiation sequence), S- $\Delta$ RS-HA (human codon usage adapted, HA-tagged and retention-signal-deleted CoV-2 S ORF), IRES (encephalomyocarditis virus internal ribosomal entry site), Puro (puromycin-resistant gene ORF), WPRE (woodchuck hepatitis virus posttranslational regulatory element), $3^{\prime}$ LTR $\Delta$ U3 (truncated HIV-1 3' long terminal repeat; self-inactivation), SV40 early pA (simian virus 40 early polyadenylation signal), Amp (ampicillin resistance gene, comprising the promoter and ORF) and pUC ori (high-copy-number pUC origin of replication). (B) Western immunoblotting of pLV-CMV-(S- $\triangle$ RS-HA)-IRES-Puro-WPRE transfected HEK293T cell protein extracts (S- $\triangle$ RS-HA; 40 and $20 \mu \mathrm{g}$ ) and pEGFP-C1 transfected HEK293T cell protein extract (GFP; $40 \mu \mathrm{g})$ employed as a negative control (-). Cross-reactive bands in both lanes indicate the loading. (C) Representative microscopic image (phase contrast, fluorescence, and merged fields; 10X) of syncytia, generated by cotransfection of HEK/ACE2/TMPRRS2/Puro cells with pLV-CMV-(S- $\triangle$ RS-HA)-IRES-Puro-WPRE construct and PEGFP-C1. (D) Representative microscopic image (phase contrast, fluorescence, and merged fields; 10X) of syncytia, generated by cocultivation of HEK/ACE2/TMPRRS2/Puro cells with pLV-CMV-(S- $\triangle$ RS-HA)-IRES-Puro-WPRE and pEGFP-C1 cotransiently transfected HEK293T cells.

\subsection{Generation of a Lentivirus-Based Pseudovirus}

Relying on the promising results obtained with pLV-CMV-(S- $\triangle$ RS-HA)-IRES-PuroWPRE construct and HEK/ACE2/TMPRRS2/Puro cell line, the reconstitution of a secondgeneration replicating incompetent lentivirus pseudotyped with CoV-2 S was attempted. The pLV-EF1 $\alpha$-(turboGFP-Luc2)-WPRE transfer vector, the p8.74 packaging vector, and the pLV-CMV-(S- $\triangle$ RS-HA)-IRES-Puro-WPRE pseudotyping vector were cotransfected in 
HEK293T cells. Twenty-four and forty-eight hours after transfection, transfected cell supernatant (TCS) was harvested, clarified, filtered, aliquoted, stored at $-80^{\circ} \mathrm{C}$, and subsequently tested on HEK/ACE2/TMPRRS2/Puro cells and on HEK293T cells as negative control. As pLV-EF1 $\alpha$-(turboGFP-Luc2)-WPRE transfer vector could simultaneously deliver two reporter genes, turboGFP and a human codon usage adapted luciferase (Luc2), cell transduction could be detected either by fluorescence or luminometry. In fact, when different amounts of TCS were tested on different numbers of HEK/ACE2/TMPRRS2/Puro cells, efficient cell transduction could be observed, but not on HEK293T negative control cells (Figure 2A-C).

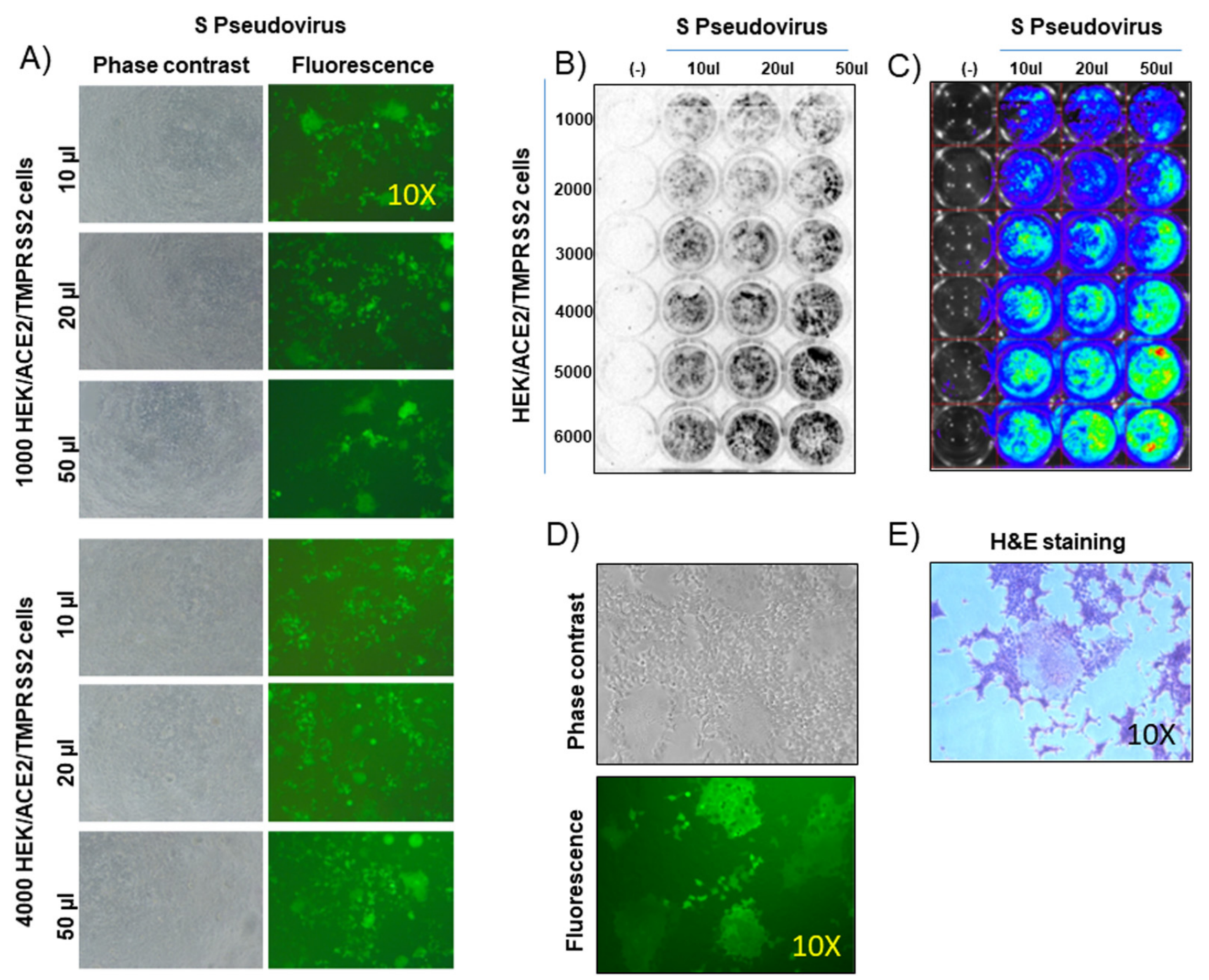

Figure 2. Pseudovirus assembly and transduction. (A) Representative images (phase contrast and fluorescence; 10X) of HEK/ACE2/TMPRRS2/Puro cells, at different numbers (1000 and 4000), transduced with different amounts $(50,20$, and $10 \mu \mathrm{L})$ of supernatant containing pseudovirus. (B) ChemiDoc and (C) IVIS luminometric detection of HEK/ACE2/TMPRRS2/Puro cells, at different numbers (1000, 2000, 3000, 4000, 5000, and 6000), transduced with different amounts $(50,20$, and $10 \mu \mathrm{L}$ ) of supernatant containing pseudovirus (S pseudovirus). Negative control was established with HEK293T cells, at different numbers $(1000,2000,3000,4000,5000$, and 6000) and transduced with $50 \mu \mathrm{L}$ of the same supernatant containing pseudovirus (S pseudovirus). (D) Representative images of syncytia (phase contrast and fluorescence of the same field; 10X), generated by pseudovirus-transduced HEK/ACE2/TMPRRS2/Puro cells. (E) Representative images of a syncytium (phase contrast; 10X) generated by pseudovirus-transduced HEK/ACE2/TMPRRS2/Puro cells and stained with hematoxylin and eosin (H\&E staining). 
Further, bona fide fusogenic activity through syncytia formation could be observed (Figure 2D,E), recapitulating natural CoV-2 infection. Syncytia formation could be considered a signature of $\mathrm{CoV}$ infection and pathogenicity as it can be observed not only in vitro but also in vivo, in lung tissue from people infected with different CoVs, such as SARS-CoV, MERS-CoV, or SARS-CoV-2 [20-25].

\subsection{Lentivirus-Based Vector Can Be Pseudotyped with ACE2/TMPRSS2}

TMPRSS2 is a serine protease with a class II transmembrane domain, whereas ACE2 is a carboxypeptidase with a class I transmembrane domain, and both of them are localized on the cell membrane surface [8] (Figure S4). Based on this information, it was reasoned that lentiviral vector particles could potentially be pseudotyped with ACE2/TMPRSS2 and allow pseudovirus penetration into cells expressing CoV-2 S. The pLV-EF1 $\alpha$-(turboGFP-Luc2)WPRE transfer vector, the p8.74 packaging vector, and the pEF1 $\alpha$-ACE2/TMPRRS2/Puro pseudotyping vector were cotransfected in HEK293T cells. Twenty-four and forty-eight hours after transfection, TCS was harvested, aliquoted, stored at $-80{ }^{\circ} \mathrm{C}$, and subsequently tested on pLV-CMV-(S- $\triangle$ RS-HA)-IRES-Puro-WPRE transiently or stably transfected HEK/S$\triangle$ RS-HA/Puro cells and on HEK293T cells as negative control. TCS transduction could be observed on HEK/S- $\triangle$ RS-HA/Puro cells but not on HEK293T negative control cells (Figure 3A,B).

Therefore, a lentivirus-based vector could be pseudotyped with ACE2/TMPRSS2. Worthy of note, this kind of pseudotypization and cell transduction did not generate syncytia as observed for the lentivirus-based vector pseudotyped with CoV-2 S. Although the reason for this issue was not investigated, because such an investigation would be outside the scope of this work, it could be assumed that the syncytia formation is an active process in part dependent on a specific intracellular apparatus, contacting the long cytoplasmic tail of ACE2 and/or TMPRSS2, that is present inside the cells but absent inside the pseudotyped lentiviral particles. It was shown that infection with CoV-2 was significantly decreased in cells expressing ACE2 mutant versions lacking the cytoplasmic domain [26].

\subsection{Assessment of a Pseudovirus Neutralization Assay}

Starting from the availability of a pseudovirus mimicking the interaction of SARSCoV-2 with target cells, it was of interest to assess a pseudovirus neutralization assay, to be applied for several purposes. The aim of this assay is to overcome the barriers presented by the complexities of other neutralization assays, such as multiple passages and the addition and removal of reagents and solutions, mainly during sera dilution and detection steps, where cells could detach from the microplate surface and serious errors could be introduced. In our simplified system, reagents and solution are only added at each step, while washing steps are absent, from the beginning to the end of the assay.

Clear-flat-bottom 96-well microplates with opaque walls were employed (Figure S5). Opaque walls prevent well-to-well crosstalk for luminometric detection, whereas clearflat-bottom wells allow direct microscopic monitoring of cell growth and/or fluorescence microscope observation of transduced cells expressing a fluorescent reporter gene, in this specific case turboGFP. The neutralization assay was performed with human sera coming from SARS-CoV-2-infected patients, collected after symptom onset, that produced positive results in PCR and ELISA tests. Sera collected prior to the SARS-CoV-2 emergence (1999) that tested negative in ELISA were used as negative control. First, serial dilutions of the sera (plasma from EDTA-treated blood can be used too) were incubated for 90 min with TCS containing pseudovirus sufficient to achieve approximately $10^{4}$ relative light units (RLUs) of luciferase signal per well (usually no more than 2 to $10 \mu \mathrm{L}$ ). Subsequently, $10^{4}$ HEK/ACE2/TMPRRS2/Puro cells were added to each well (Figure S5). As previously mentioned, HEK/ACE2/TMPRRS2/Puro cells do not need to be constantly kept in culture under puromycin selection: a large batch of them can be grown, aliquoted at a suitable concentration, and stored at $-80{ }^{\circ} \mathrm{C}$ or in liquid nitrogen and be immediately ready to use after thawing. Alternatively, HEK293T cells can be electroporated with pEF1 $\alpha-$ 
ACE2/TMPRRS2/Puro and directly used, obtaining the same result. NT50 could be measured as soon as $48 \mathrm{~h}$ after adding the cells, either by a fluorescence microscope or by luminometry and simply adding luciferin in each well (Figure S5 and Figure 4A,B), without the need for cell lysis and transferring the lysate to a different microplate. Moreover, light detection can be performed with a digital imager for Western blotting, BLI, or by a multiwell plate reader luminometer.

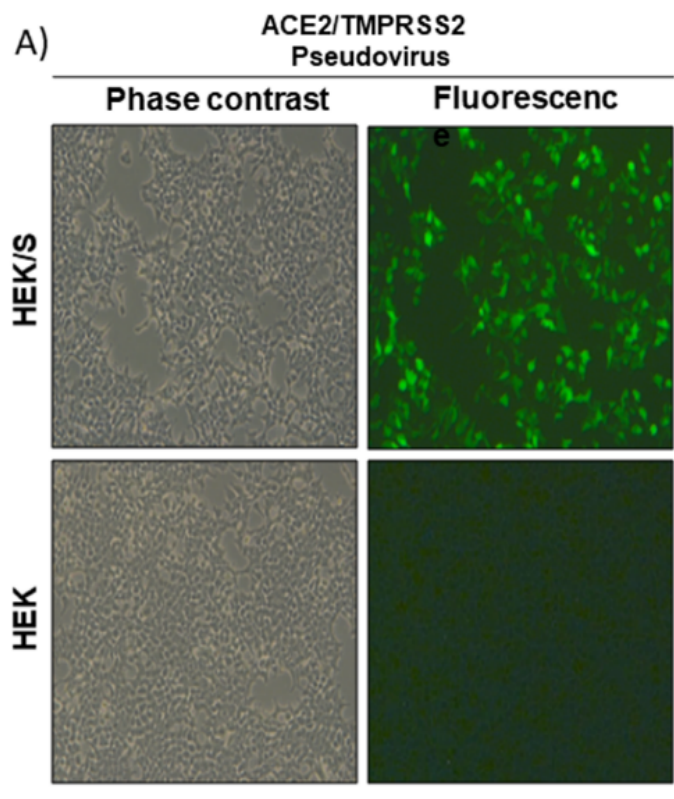

C) Cell surface Pseudovirus surface

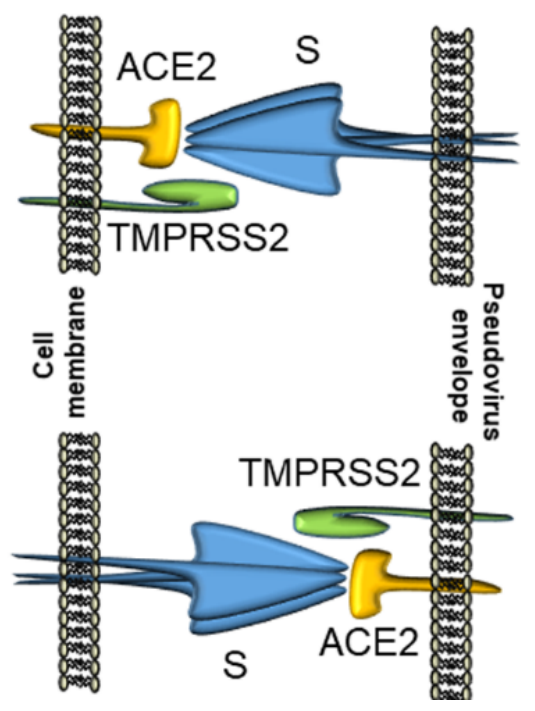

\section{B) $\begin{array}{r}\text { ACE2/TMPRSS2 } \\ \text { Pseudovirus }\end{array}$}

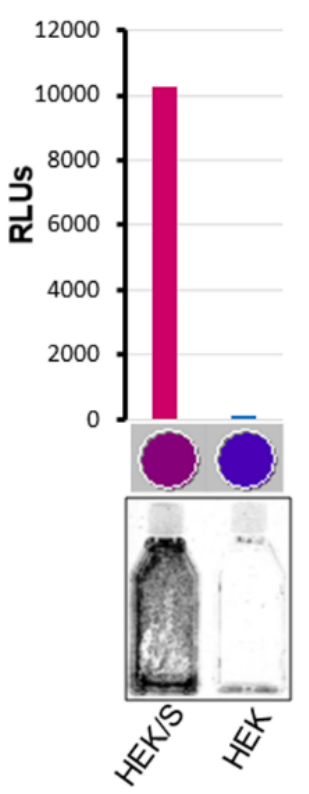

Syncytia formation

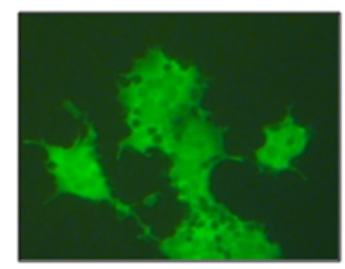

No syncytia formation

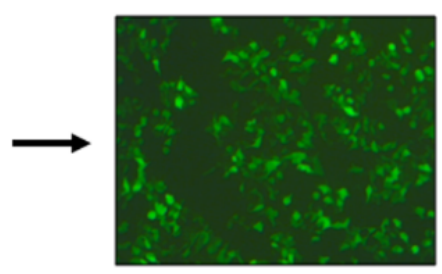

Figure 3. Pseudovirus generation with ACE2 and TMPRSS2. (A) Representative image (phase contrast and fluorescence of the same field; 10X) of HEK/S-DRS-HA/Puro cells (HEK/S) and HEK293T cells (HEK) transduced with ACE2/TMPRSS2 pseudovirus. (B) Representative $25 \mathrm{~cm}^{2}$ flasks of HEK/S- $\triangle$ RS-HA/Puro cells (HEK/S) and HEK293T cells (HEK) transduced with ACE2/TMPRSS2 pseudovirus and exposed to the ChemiDoc apparatus. Luminometric quantification of the lysate of the same cells, expressed as relative luciferase units (RLUs) and colorimetrically visualized (violet and blue spots) by the luminometer software, during and after reading. (C) Drawing explaining the lack of syncytia generation by ACE2/TMPRSS2 pseudovirus. 


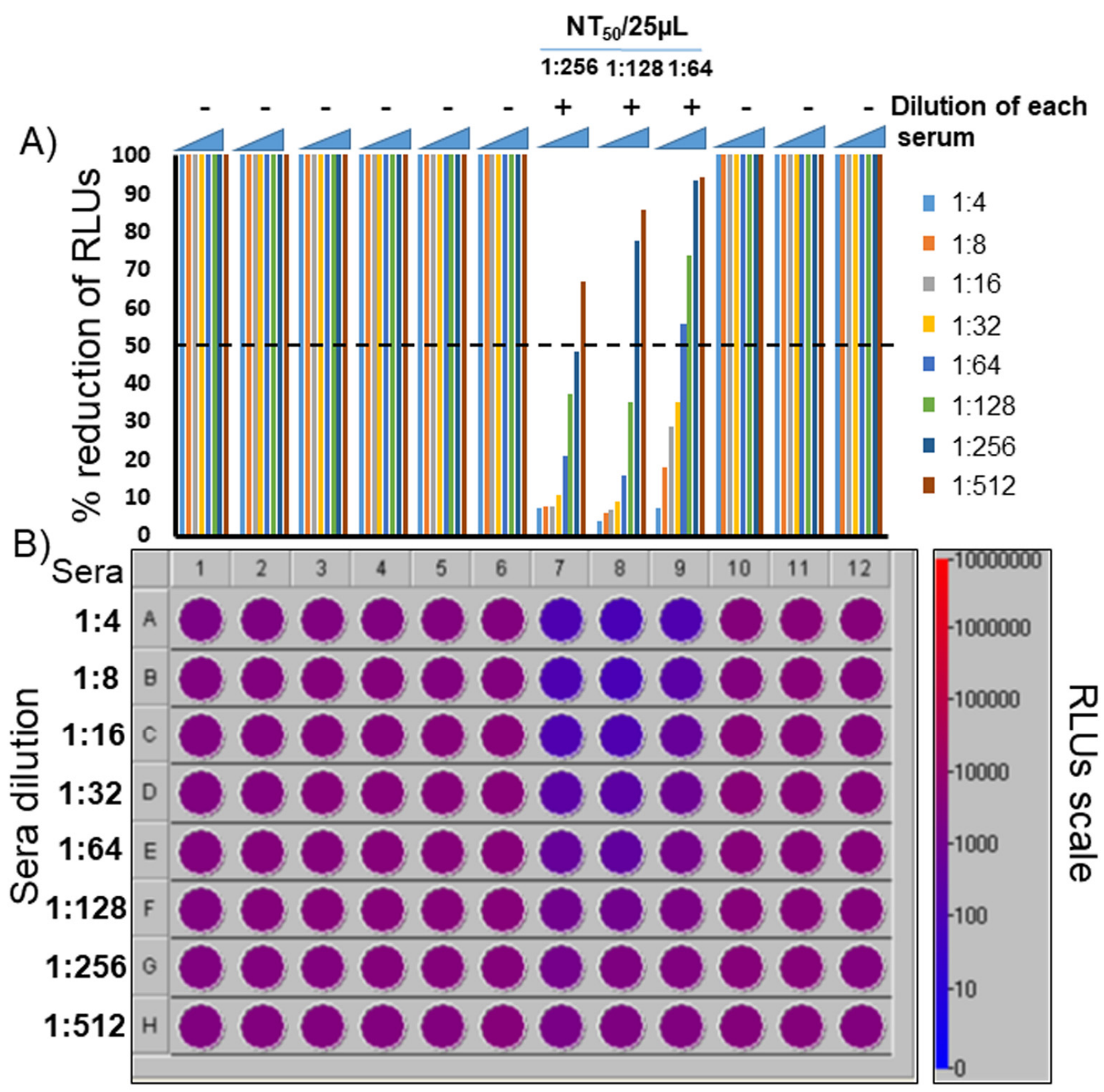

Figure 4. Representative pseudovirus neutralization assay. Six PCR and ELISA CoV-2 negative patients' representative sera (1 to 6), three PCR and ELISA CoV-2 positive patients' sera (7 to 9), and two pre-pandemic sera (collected in 1998; 10 and 11) were tested. A further negative control was established without serum (12), which was substituted with complete medium. (A) Histogram obtained from luminometric detection of transduced cells with pseudovirus. Relative luciferase units (RLUs) were compared and normalized to those derived from wells where pseudovirus was added in the absence of sera ( $100 \%)$. Neutralization titer $50\left(\mathrm{NT}_{50}\right)$ was expressed as the maximal dilution of the sera where the reduction of the signal was $\geq 50 \%$. In this specific example, the $\mathrm{NT}_{50} / 25 \mu \mathrm{L}$ of serum 7 is 1:256, that of serum 8 is 1:128, and that of serum 9 is $1: 64$. However, because the titer was measured at $25 \mu \mathrm{L}$, the apparent $\mathrm{NT}_{50}$ has to be multiplied by 40 to obtain the normalized $\mathrm{NT}_{50} / \mathrm{mL}$. Therefore, the $\mathrm{NT}_{50} / \mathrm{mL}$ of the sera 7 is $1: 10,256$, that of the serum 8 is 1:5120, and that of the serum 9 is $1: 2560$. Bar colors indicate sera dilution. (B) Colorimetric visualization of the RLUs displayed by the luminometer software, during and after reading.

Thus, a rapid, flexible, reliable, sensitive, and cost-effective pseudovirus neutralization assay for SARS-CoV-2 was established, where all steps could be performed in the same plate and in a standard BSL2 laboratory, as the pseudovirus is completely safe. With this assay, we have been able to rapidly and cheaply determine the neutralizing potency of mAbs 
derived from serum or plasma samples in a BSL2 laboratory. Automation and additional miniaturization to further increase throughput are certainly possible. As serum neutralizing activity has long been identified as a correlate of protection in many viral infections [27], including CoV infections [28], and a global CoV-2 vaccination campaign is ongoing, it is absolutely important to have a practical test to assess the outcome of vaccination. In order to meet this need, such a test must be rapid, flexible, reliable, sensitive, quantitative, cost-effective, safe, and easily adapted to different coronavirus variants $[29,30]$.

Supplementary Materials: The following are available online at https:/ / www.mdpi.com/article/10 .3390/vaccines9040389/s1. Figure S1: ACE2/TMPRSS2 expressing construct.; Figure S2: S- $\Delta$ RS-HA human adapted ORF sequence and Biscistronic turboGFP-IRES-Luc2 ORF; Figure S3: pLV-CMV-(S$\triangle$ RS-HA)-IRES-Puro-WPRE and representive phase contrast images (10X) of healthy donor serum treated pLV-CMV-(S- $\triangle$ RS-HA)-IRES-Puro-WPRE transfected HEK/ACE2/TMPRRS2/Puro cells (large syncytia formation) and COVID-19 convalescent serum treated pLV-CMV-(S- $\triangle$ RS-HA)-IRESPuro-WPRE transfected HEK/ACE2/TMPRRS2/Puro cells (absence of syncytia).; Figure S4: ACE2 and TMPRSS2 topology; Figure S5: Serum Neutralization Flow-chart.

Author Contributions: Conceptualization, G.D.; methodology, G.D., V.F., F.M. and L.R.; resources, G.D., A.R., V.M., F.C., N.G., C.F. and G.M.; writing-original draft preparation, G.D.; writing, G.D.; review and editing, all authors; project administration, G.D.; funding acquisition, G.D. All authors have read and agreed to the published version of the manuscript.

Funding: This research received no external funding.

Institutional Review Board Statement: Not applicable.

Informed Consent Statement: Not applicable.

Data Availability Statement: Data is contained within the article. Reported results can be found in Supplementary Materials.

Acknowledgments: We would like to thank all the people who decided to donate their blood for the study and Diletta Laccabue, Laboratory of Viral Immunopathology, Unit of Infectious Diseases and Hepatology, Azienda Ospedaliero-Universitaria di Parma, for taking care of the experimental protocol submission to the ethical committee.

Conflicts of Interest: No potential conflict of interest was reported by the authors.

\section{References}

1. Han, Q.; Lin, Q.; Jin, S.; You, L. Coronavirus 2019-nCoV: A brief perspective from the front line. J. Infect. 2020, 80, 373-377. [CrossRef] [PubMed]

2. Callaway, E. The race for coronavirus vaccines: A graphical guide. Nature 2020, 580, 576-577. [CrossRef] [PubMed]

3. Poland, G.A.; Ovsyannikova, I.G.; Kennedy, R.B. SARS-CoV-2 immunity: Review and applications to phase 3 vaccine candidates. Lancet 2020, 396, 1595-1606. [CrossRef]

4. Iacob, S.; Iacob, D.G. SARS-CoV-2 Treatment Approaches: Numerous Options, No Certainty for a Versatile Virus. Front. Pharmacol. 2020, 11, 1224. [CrossRef]

5. Li, G.; De Clercq, E. Therapeutic options for the 2019 novel coronavirus (2019-nCoV). Nat. Rev. Drug Discov. 2020, 19, 149-150. [CrossRef]

6. Bonam, S.R.; Kotla, N.G.; Bohara, R.A.; Rochev, Y.; Webster, T.J.; Bayry, J. Potential immuno-nanomedicine strategies to fight COVID-19 like pulmonary infections. Nano Today 2021, 36, 101051. [CrossRef]

7. Walls, A.C.; Park, Y.J.; Tortorici, M.A.; Wall, A.; McGuire, A.T.; Veesler, D. Structure, Function, and Antigenicity of the SARS-CoV-2 Spike Glycoprotein. Cell 2020, 181, 281-292.e286. [CrossRef]

8. Hoffmann, M.; Kleine-Weber, H.; Schroeder, S.; Kruger, N.; Herrler, T.; Erichsen, S.; Schiergens, T.S.; Herrler, G.; Wu, N.H.; Nitsche, A.; et al. SARS-CoV-2 Cell Entry Depends on ACE2 and TMPRSS2 and Is Blocked by a Clinically Proven Protease Inhibitor. Cell 2020, 181, 271-280e278. [CrossRef]

9. Yan, R.; Zhang, Y.; Li, Y.; Xia, L.; Guo, Y.; Zhou, Q. Structural basis for the recognition of SARS-CoV-2 by full-length human ACE2. Science 2020, 367, 1444-1448. [CrossRef]

10. Lin, K.; Liu, M.; Ma, H.; Pan, S.; Qiao, H.; Gao, H. Laboratory biosafety emergency management for SARS-CoV-2. J. Biosaf. Biosecur. 2020. [CrossRef] 
11. Case, J.B.; Rothlauf, P.W.; Chen, R.E.; Liu, Z.; Zhao, H.; Kim, A.S.; Bloyet, L.M.; Zeng, Q.; Tahan, S.; Droit, L.; et al. Neutralizing Antibody and Soluble ACE2 Inhibition of a Replication-Competent VSV-SARS-CoV-2 and a Clinical Isolate of SARS-CoV-2. Cell Host Microbe 2020, 28, 475-485.e475. [CrossRef]

12. Nie, J.; Li, Q.; Wu, J.; Zhao, C.; Hao, H.; Liu, H.; Zhang, L.; Nie, L.; Qin, H.; Wang, M.; et al. Establishment and validation of a pseudovirus neutralization assay for SARS-CoV-2. Emerg. Microbes Infect. 2020, 9, 680-686. [CrossRef]

13. Crawford, K.H.D.; Eguia, R.; Dingens, A.S.; Loes, A.N.; Malone, K.D.; Wolf, C.R.; Chu, H.Y.; Tortorici, M.A.; Veesler, D.; Murphy, M.; et al. Protocol and Reagents for Pseudotyping Lentiviral Particles with SARS-CoV-2 Spike Protein for Neutralization Assays. Viruses 2020, 12, 513. [CrossRef]

14. Giroglou, T.; Cinatl, J., Jr.; Rabenau, H.; Drosten, C.; Schwalbe, H.; Doerr, H.W.; von Laer, D. Retroviral vectors pseudotyped with severe acute respiratory syndrome coronavirus S protein. J. Virol. 2004, 78, 9007-9015. [CrossRef]

15. Ujike, M.; Huang, C.; Shirato, K.; Makino, S.; Taguchi, F. The contribution of the cytoplasmic retrieval signal of severe acute respiratory syndrome coronavirus to intracellular accumulation of $\mathrm{S}$ proteins and incorporation of $\mathrm{S}$ protein into virus-like particles. J. Gen. Virol. 2016, 97, 1853-1864. [CrossRef]

16. Jouvenet, N.; Neil, S.J.D.; Bess, C.; Johnson, M.C.; Virgen, C.A.; Simon, S.M.; Bieniasz, P.D. Plasma membrane is the site of productive HIV-1 particle assembly. PLoS Biol. 2006, 4, e435. [CrossRef]

17. Gustafsson, C.; Govindarajan, S.; Minshull, J. Codon bias and heterologous protein expression. Trends Biotechnol. 2004, $22,346-353$. [CrossRef]

18. Kudla, G.; Lipinski, L.; Caffin, F.; Helwak, A.; Zylicz, M. High guanine and cytosine content increases mRNA levels in mammalian cells. PLoS Biol. 2006, 4, e180. [CrossRef]

19. Ziegler, C.G.K.; Allon, S.J.; Nyquist, S.K.; Mbano, I.M.; Miao, V.N.; Tzouanas, C.N.; Cao, Y.; Yousif, A.S.; Bals, J.; Hauser, B.M.; et al. SARS-CoV-2 Receptor ACE2 Is an Interferon-Stimulated Gene in Human Airway Epithelial Cells and Is Detected in Specific Cell Subsets across Tissues. Cell 2020, 181, 1016-1035.e1019. [CrossRef]

20. Buchrieser, J.; Dufloo, J.; Hubert, M.; Monel, B.; Planas, D.; Rajah, M.M.; Planchais, C.; Porrot, F.; Guivel-Benhassine, F.; Van der Werf, S.; et al. Syncytia formation by SARS-CoV-2-infected cells. EMBO J. 2020, e106267. [CrossRef]

21. Bussani, R.; Schneider, E.; Zentilin, L.; Collesi, C.; Ali, H.; Braga, L.; Volpe, M.C.; Colliva, A.; Zanconati, F.; Berlot, G.; et al. Persistence of viral RNA, pneumocyte syncytia and thrombosis are hallmarks of advanced COVID-19 pathology. EBioMedicine 2020, 61, 103104. [CrossRef]

22. Franks, T.J.; Chong, P.Y.; Chui, P.; Galvin, J.R.; Lourens, R.M.; Reid, A.H.; Selbs, E.; McEvoy, C.P.; Hayden, C.D.; Fukuoka, J.; et al. Lung pathology of severe acute respiratory syndrome (SARS): A study of 8 autopsy cases from Singapore. Hum. Pathol. 2003, 34, 743-748. [CrossRef]

23. Xia, S.; Liu, M.; Wang, C.; Xu, W.; Lan, Q.; Feng, S.; Qi, F.; Bao, L.; Du, L.; Liu, S.; et al. Inhibition of SARS-CoV-2 (previously 2019-nCoV) infection by a highly potent pan-coronavirus fusion inhibitor targeting its spike protein that harbors a high capacity to mediate membrane fusion. Cell Res. 2020, 30, 343-355. [CrossRef]

24. Matsuyama, S.; Nagata, N.; Shirato, K.; Kawase, M.; Takeda, M.; Taguchi, F. Efficient activation of the severe acute respiratory syndrome coronavirus spike protein by the transmembrane protease TMPRSS2. J. Virol. 2010, 84, 12658-12664. [CrossRef]

25. Qian, Z.; Dominguez, S.R.; Holmes, K.V. Role of the spike glycoprotein of human Middle East respiratory syndrome coronavirus (MERS-CoV) in virus entry and syncytia formation. PLoS ONE 2013, 8, e76469. [CrossRef]

26. Haga, S.; Yamamoto, N.; Nakai-Murakami, C.; Osawa, Y.; Tokunaga, K.; Sata, T.; Yamamoto, N.; Sasazuki, T.; Ishizaka, Y. Modulation of TNF-alpha-converting enzyme by the spike protein of SARS-CoV and ACE2 induces TNF-alpha production and facilitates viral entry. Proc. Natl. Acad. Sci. USA 2008, 105, 7809-7814. [CrossRef] [PubMed]

27. Plotkin, S.A. Correlates of Protection Induced by Vaccination. Clin. Vaccine Immunol. 2010, 17, 1055-1065. [CrossRef] [PubMed]

28. Kellam, P.; Barclay, W. The dynamics of humoral immune responses following SARS-CoV-2 infection and the potential for reinfection. J. Gen. Virol. 2020, 101, 791-797. [CrossRef]

29. Wang, P.; Nair, M.S.; Liu, L.; Iketani, S.; Luo, Y.; Guo, Y.; Wang, M.; Yu, J.; Zhang, B.; Kwong, P.D.; et al. Increased Resistance of SARS-CoV-2 Variants B.1.351 and B.1.1.7 to Antibody Neutralization. bioRxiv 2021. [CrossRef]

30. Wang, P.; Nair, M.S.; Liu, L.; Iketani, S.; Luo, Y.; Guo, Y.; Wang, M.; Yu, J.; Zhang, B.; Kwong, P.D.; et al. Antibody Resistance of SARS-CoV-2 Variants B.1.351 and B.1.1.7. Nature 2021. [CrossRef] 